\title{
Real-time single-pass connected components analysis algorithm
}

\author{
Fei Zhao ${ }^{*}$, Huan zhang Lu and Zhi yong Zhang
}

\begin{abstract}
Due to the demand for real-time processing in real-time automatic target recognition (RTATR) systems, fast connected components analysis (CCA) is significant to RTATR performance improvement. Conventional single-pass CCA algorithms need horizontal blanking periods to resolve the equivalence, which are difficult to be applied when the streamed data is transmitted without horizontal blanking periods. In this paper, a real-time single-pass CCA algorithm is proposed. Unlike the conventional ones, we adopt the pixel as a scan unit while the line as a labeling unit and manage the correspondence of labels between adjacent rows by designing a multi-layer-index structure. Equivalence is resolved when the image is scanning, without extra processing time. The proposed algorithm is suitable for hardware acceleration, and the streamed image data can be processed during image transmission without horizontal blanking periods. Experimental results indicate that the hardware acceleration of algorithm achieves real-time CCA in RTATR system.
\end{abstract}

Keywords: Connected components analysis, Real-time processing, Single pass, Hardware acceleration

\section{Introduction}

Connected components analysis (CCA) is an important step in many machine vision applications. In real-time automatic target recognition (RTATR) systems, such as imaging homing guidance weapon systems, CCA is one of the most fundamental operations, which extracts features of targets for tracking and recognition. Because of the high-frame rate, which is usually no less than $60 \mathrm{~Hz}$, only about $17 \mathrm{~ms}$ are allowed in each frame for all the tasks (such as target detection, tracking, and recognition), and less time for fundamental processing. Therefore, fast CCA is significant to the performance improvement in these systems. To date, two main lines of CCA have been followed in general:

(1)Labeling first algorithms [1-5]. These algorithms label the binary image first, and then the features are extracted from the labeled image. The connected components labeling is a general purpose method, and many researchers have concentrated on the fast connected components labeling. Existing fast connected components labeling (CCL) algorithm can be divided into two classes: (a) labelequivalence-based algorithms [1-4] and (b) regiongrowing-based algorithms [5]. These algorithms process an image in the raster-scan order (top to bottom, left to right) at least twice. In the first scan, provisional labels are assigned, and then the key point is to resolve label equivalence by finding a unique representative label for each group of equivalent labels. In the second scan, the pixel's provisional label is replaced by the representative label. According to different scan unit, labelequivalence-based algorithms can be divided into run-based algorithms [1], pixel-based algorithms [2,4], and block-based algorithms [3]. Label equivalence is not recorded and resolved in such algorithms, and connected components of any shapes can be labeled in single scan. But these algorithms access the image in an irregular way, which means that the whole image must be available before labeling.

\footnotetext{
* Correspondence: f_z2010@126.com

National Key Laboratory of Automatic Target Recognition (ATR), National

University of Defense Technology, Changsha, Hunan, Peoples' Republic of China
}

\section{Springer}

(c) 2013 Zhao et al.; licensee Springer. This is an Open Access article distributed under the terms of the Creative Commons Attribution License (http://creativecommons.org/licenses/by/2.0), which permits unrestricted use, distribution, and reproduction in any medium, provided the original work is properly cited. 
Most of the above-mentioned algorithms were designed or improved on general purpose processor (GPP) platform (e.g., PC), and some of them have achieved a good performance. For example, algorithms in [1] and [5] offered higher processing speed than others and they only need less than $4 \mathrm{~ms}$ to label $512 \times 512$ binary images on highperformance PC (Intel Pentium D 3.0 GHz + 3.0 GHz CPUs, 2 GB memory, Mandriva Linux OS; Intel Corporation, Sta. Clara, CA, USA) [1]. However, these fast algorithms still cannot meet the real-time processing demand in the RTATR systems. This is because the processors' performance in RTATR system is far poorer than the PC mentioned above when power consumption and volume must be considered. Therefore, studies on algorithms which can be easily accelerated by hardware (e.g., implemented on field-programmable gate array, FPGA) become a feasible way to figure out real-time problem. Crookes, Benkrid, et al. have implemented a resourceefficient multi-pass algorithm on FPGA [6], but indeterminate number of passes is required to complete the labeling. Jablonski and Gorgon [7] have implemented the classic two-pass connected components labeling on FPGA, but the method requires a second pass, and twoclock cycles per pixel plus a small overhead for region merging. Kofi et al. have presented a run-length-based connected component algorithm for FPGA implementation $[8,9]$, runs in images are extracted and initially labeled in the first pass, then equivalence sets are resolved, and runs are translated to connected components. However, the equivalence resolution is still sequential and requires that all runs are initially labeled at first. Clearly, it is unsuitable for real-time processing of streamed image data.

(2) Single-pass CCA algorithms [10-12]. D.G. Baily et al. have proposed another CCA method, in a more goal-directed way: Single-pass CCA algorithm, which accumulates the feature data (such as the area, center of gravity, and perimeter) while the pixels are being scanned and labeled, does not generate a labeled image. Essentially, the single-pass CCA algorithms are label-equivalence-based too, which resolve equivalence in each row during the horizontal blanking periods as well as merge accumulated data. These algorithms eliminate the need for producing a labeled image and spare the second re-labeling pass, hence suitable for processing streamed image data on FPGA. Furthermore, an optimized single-pass CCA algorithm [12] is proposed, which applies a label recycling scheme between adjacent rows to save the memory resources. Such methods will be of great benefit to the RTATR systems: streamed images can be processed in the transmission from detector to processing unit and this will save the processing time and shorten the delays of closed-loop control. However, these methods require horizontal blanking periods to resolve equivalence, which will be a bottleneck when the streamed data is transmitted without horizontal blanking periods.

In this study, we present a new single-pass CCA algorithm, which eliminates the requirement for horizontal blanking periods in aforementioned methods, and the FPGA implementation of proposed algorithm which achieves real-time processing for streamed images. The remainder of this paper is organized as follows. Section 2 presents the principle of the algorithm. In Section 3, the hardware implementation of the proposed method is introduced. Section 4 gives the experiment results of the algorithm in both $\mathrm{PC}$ and RTATR platforms, and the comparison with existing single-pass CCA algorithm is presented, too. Section 5 concludes the discussion.

\section{Proposed algorithm}

Classical label-equivalence-based algorithms set pixel as a scan unit, so redundant labels and label equivalence will be generated for stair-like connected components. As shown in Figure 1 (assuming four-connectivity), the pixels belonging to the same region are labeled with different numbers, and more label equivalence will be encountered while the image is being scanned (the shadowed squares represent the pixels at which label equivalence is encountered). It also means that more

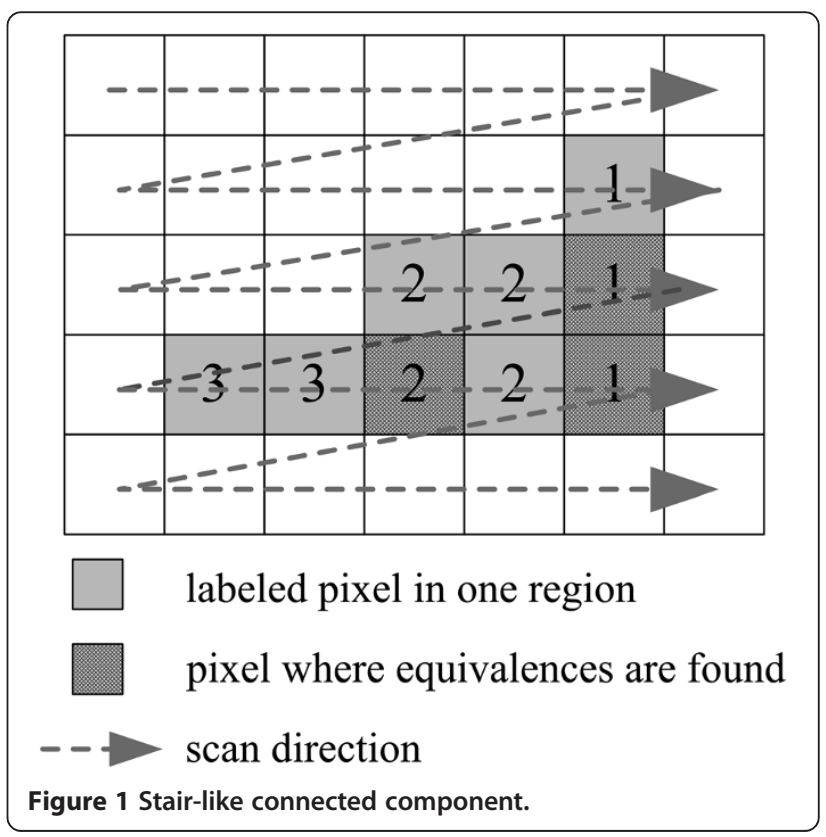


resources are needed for equivalence resolving. Runbased algorithms can solve the problem, and runs extraction is necessary in such algorithm. When a run is extracted, connectivity of runs between adjacent rows is detected, and label equivalence is recorded and resolved. In other words, few operations are needed before a run is extracted, and most operations are gathered at the end of a run. Centralized operations become the bottleneck of hardware acceleration.

In this paper, we combine the advantages of pixelbased and run-based algorithms, adopt pixel as a scan unit and run as a labeling unit. That means we scan the image pixel by pixel without labeling, and the labels are assigned for the runs. Therefore, redundant labels and equivalence shown in Figure 1 are avoided, the operations are distributed to each pixel, and only run labels assigned in the previous row are concerned in labeling and equivalence. In addition, a multi-layer-index structure is designed to manage the correspondence between labels in the previous row and labels in the current row. The structure of multi-layer-index is shown in Figure 2.

As shown in Figure 2, Previous_P_Label and New_P Label are provisional labels assigned in the previous row and the current row; Previous_F_Label and New_F_Label are label-index tables, which indicate the representative labels corresponding to Previous_P_Label and New_P Label. For instance, if Previous_F_Label $(p)=n$, it indicates that the representative label for $p$ is $n$, in which $p$ and $n$ are labels assigned in the previous row; if Previous_F_Label $(p)=0$, it indicates that the representative label for $p$ is itself, and New_F_Label has the same meaning in the current row. MAP is the translation table, which denotes the correspondence between the representative labels in the previous row and the provisional labels in the current

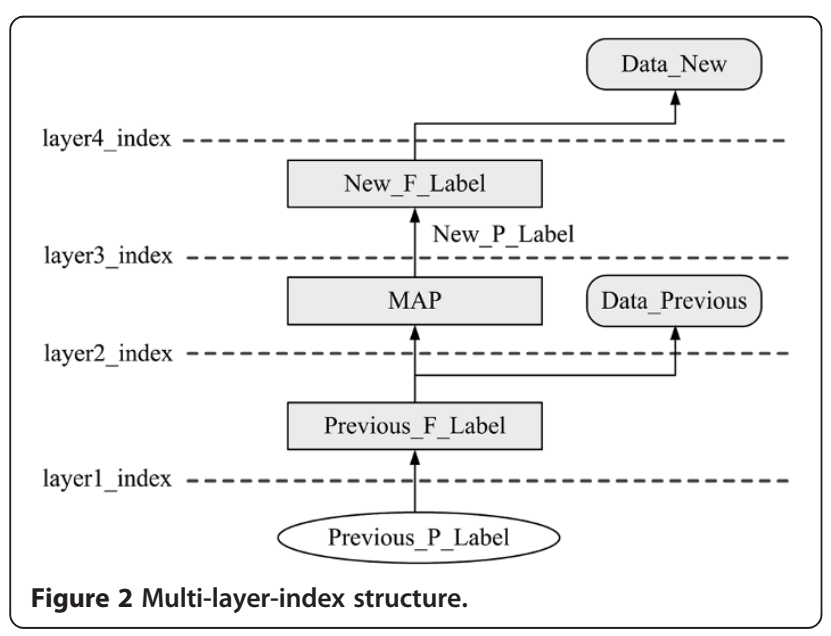

row. If $\operatorname{MAP}(i)=k$, it indicates that $i$ which is a representative label in the previous row has been translated to label $k$ in the current row; if $\operatorname{MAP}(i)=0$, it indicates that representative label $i$ in the previous row does not have a translated label in the current row. The feature data tables which store the accumulated features are presented as Data_Previous and Data_New and the four layer indexes (shown as layer1_index, layer2_index, layer3_index, and layer4_index in Figure 2) form a pipeline when a provisional label in the previous row is indexed.

In our method, the key point is to keep the consistency of multi-layer-index between adjacent rows, which means the correctness of correspondence(such as correspondence between labels in adjacent rows, correspondence between feature data in adjacent rows, and correspondence between feature data and labels in the same row) must be kept in the scanning. By setting the run as labeling unit, only the labels in the previous row are concerned in the scanning. Thus, the assignment of provisional labels in the current row is simplified, and only in some special cases (such as overlapping between runs in adjacent rows, label equivalence and tail of runs) does the multi-layer-index structure need to be updated. Furthermore, the updating can be distributed into each layer of multi-layer-index (from layer1_index to layer4_index), and the operations in each layer can be accomplished independently while the pixel is in scanning. By doing this, all the special cases can be resolved in the scanning, eliminating the need for horizontal blanking periods. In the scanning of pixels, the feature data are accumulated and labels are assigned to the runs, the multi-layer-index is updated in some special cases to keep the consistency, and the features of completed connected components are passed to subsequent processing unit. In this study, the proposed algorithm is divided into two blocks for introduction: (1) special case detection and feature accumulation and (2) multi-layer-index update.

\subsection{Special case detection and feature accumulation}

In the pixel-based scan process, the run is set as labeling unit, which means we must detect the beginning of the run at first and accumulate the features before the tail of the run. When the scanning pixel lies inside the run, overlapping between runs in adjacent rows and label equivalence in the previous row are detected, and some details (presented as overlapping modes and equivalence modes) about the special cases are provided for the updating. When the scanning pixel is the tail of the run, the run which is just scanned will be labeled by a provisional label, and some details about the run (such as run end modes) will be sent to the updating module, too. 
To detect the overlapping of runs in the pixel-based scan, the relations between scanned pixels and runs in the current row need to be detected first. For a binary image, suppose that the current scanning pixel is $P(y, x)$, $y$ and $x$ are row and column. The information of pixels in the previous row $(\mathrm{y}-1)$ is stored as info $(x)=$ $\{$ run_start, $g$, label $\}$, in which $x$ is column; run_start $=1$ indicates that $P(y-1, x)$ is the head of a run, $g=1$ indicates that $P(y-1, x)$ is nonzero, label is the provisional label of run which contains $P(y-1, x)$. When $P(y, x)$ is scanned, the relation between $P(y, x)$ and runs is extracted as follows:

(1) If $P(y, x-1)=0$ and $P(y, x) \neq 0$, then $P(y, x)$ is the beginning of a run.
(2) If $P(y, x-1) \neq 0$ and $(y, x) \neq 0$, then $P(y, x)$ exists inside a run.

(3) If $P(y, x-1) \neq 0$ and $P(y, x)=0$, then $P(y, x)$ is the first pixel behind the tail of a run.

(4) If $P(y, x-1)=0$ and $P(y, x)=0$, then $P(y, x)$ does not exist inside the run.

In this paper, we take four-connectivity as example to detect the connectivity between adjacent rows. The whole flow of special case detection and label assignment is shown in Figure 3, in which overlapped is the flag of overlapping between runs in adjacent rows; when overlapped $=1$, it indicates the run which contains $P(y, x)$ is connected with the run labeled as last label in the previous row; pfl $1=$ last_label if Previous_F_Label(last_

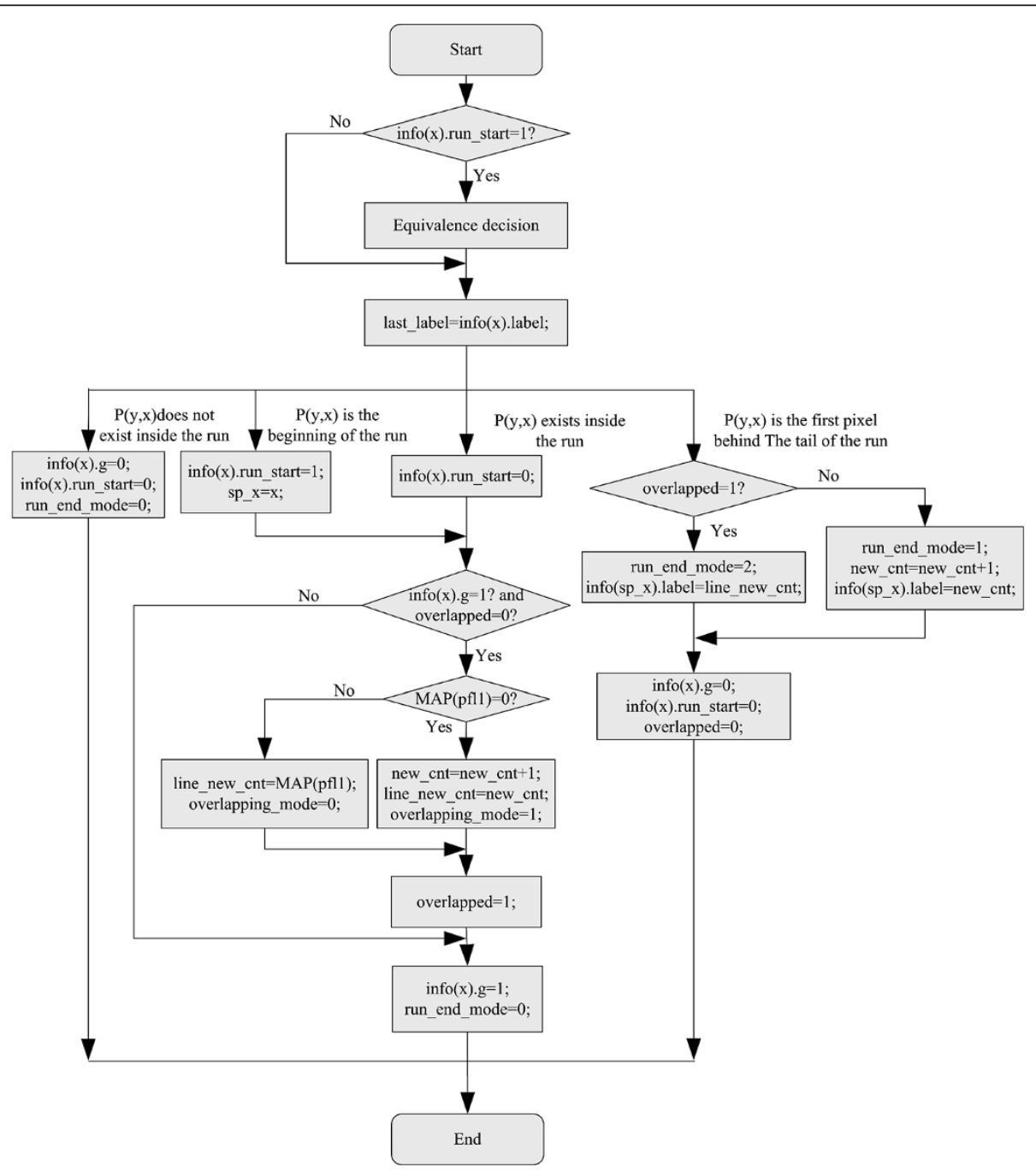

Figure 3 Special case detection and label assignment. 
label $)=0$, else pfl1 = Previous_F_Label(last_label $) ;$ new cnt indicates the assigned new provisional label in the current row.

As shown in Figure 3, when the image is scanned, the label in the previous row is stored in last_label and equivalence decision is performed. When $P(y, x)$ is the beginning of the run or inside a run, the connectivity is detected by judging info(x).g. If the connectivity is detected (overlapped $=1$ ), the translated label (the label which corresponds to $p f l 1$ in the current row (i.e., MAP $(p f l 1))$ when $\operatorname{MAP}(p f l 1) \neq 0$, or new_cnt when MAP $(p f l 1)=0)$ is stored in line_new_cnt, and it is used to assign provisional label for the run when the first pixel behind the tail of the run is encountered, the line_new_cnt is also used for updating of the multi-layer-index structure. The overlapping mode (overlapping_mode) and run end mode (run_end_mode) are also recorded for the updating of the multi-layer-index structure. In the scanning, updating of $\operatorname{info}(x) . g$ and info(x).run_start is performed at each pixel, and assignment of info $(x)$.label is performed at the tail of the run.

By setting the run as labeling unit, the equivalence is detected between two provisional labels which are assigned in the previous row. As shown in Figure 3, equivalence decision is performed when the beginning of the run in the previous row is found: when $P(y, x) \neq 0$ and info $(x)$.run_start $=1$, if overlapped $=1$, it indicates that last_label and info $(x)$.label are equivalent. Suppose
Depending on the different results of layer2_index for last_label and info $(x)$.label (i.e., different provisional labels in the current row which correspond to last_label and info $(x)$.label), two different equivalence modes (shown as equ_mode $=1$ and $e q u \_$mode $=2$ in Figure 4) may exist, which are important for multi-layer-index updating.

For analysis of the features of the region, such as the area, center of gravity, bounding box, or perimeter, some features should be collected when each pixel is scanned. When position of $P(y, x)$ is judged, the data can be accumulated from the beginning to the tail of the run, and the accumulated data can be used for updating of data table.

\subsection{Multi-layer-index update}

Depending on different modes of special cases (such as overlapping between runs in adjacent rows, equivalence and run end), the multi-layer-index structure should be updated during the scanning to maintain the correctness of index results in each layer.

\subsubsection{Overlapping between runs in adjacent rows}

To save the resources for labels storing, labels are reused between adjacent rows. When the scanning pixel $P(y, x)$ connects with run $L_{p}$ in the previous row, it means that the connected component which contains $L_{p}$ is not complete, label assigned for $L_{p}$ in the previous row should

$$
\begin{aligned}
& \text { pfl2 }=\left\{\begin{array}{l}
\text { info }(x) \cdot \text { label } \\
\text { Previous_F_Label }(\text { info }(x) \cdot \text { label }) \quad, \quad \text { if } \quad \text { Previous_F_Label }(\text { info }(x) \cdot \text { label })=0
\end{array}\right. \\
& p l 2=\operatorname{Map}(p f l 2)
\end{aligned}
$$
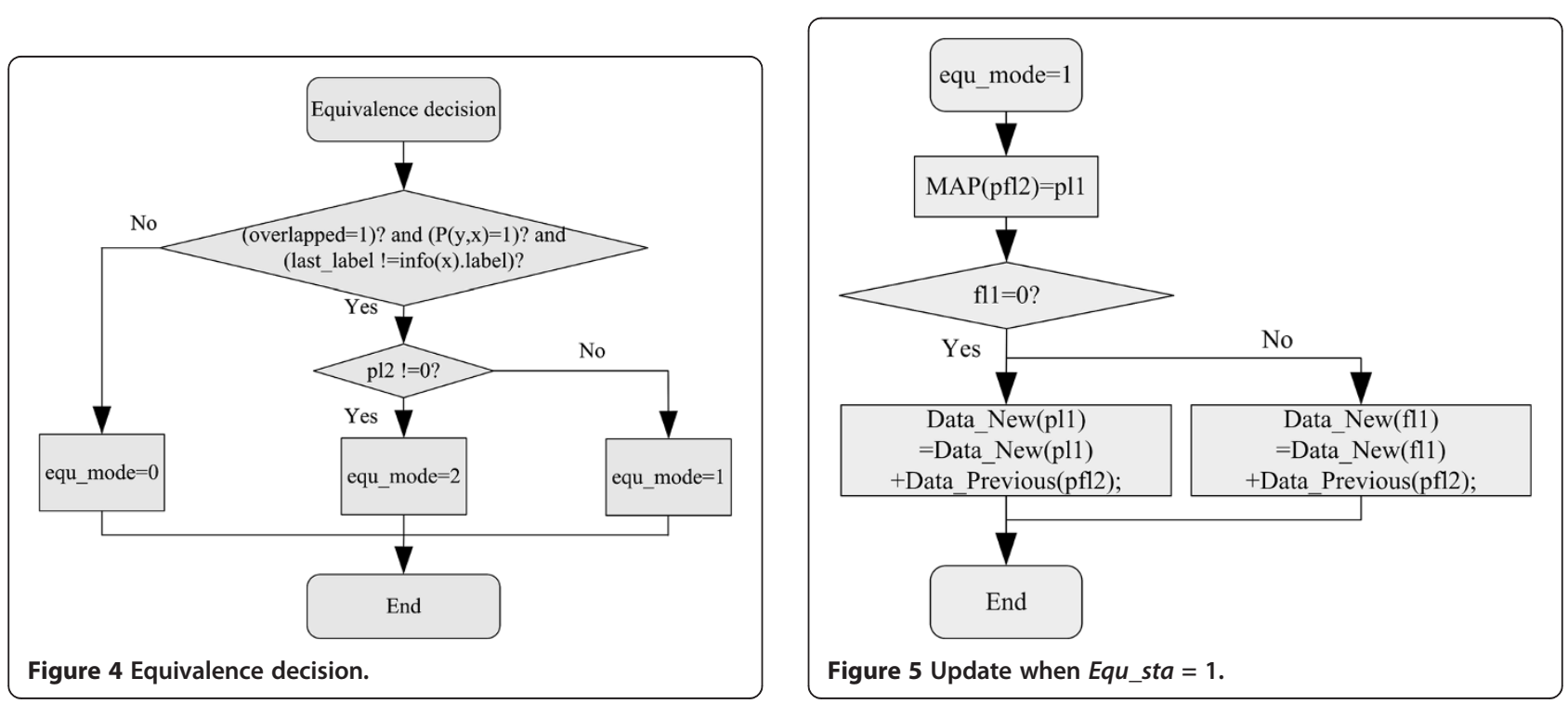
be translated to a new label, and the translation table MAP needs to be updated.

When it is the first time to set overlapped $=1$ and MAP $(p f l 1)=0$ (as shown in Figure 3) (which means $L_{p}$ connects with no pixels before, corresponding to overlapping mode $=1$ ), a new label in the current row is assigned and the translation table MAP is updated as $\mathrm{MAP}(p f l 1)=$ line_new_cnt; line_new_cnt is the assigned new label which is recorded in Figure 3. The feature data is translated as Data_New (line_new_cnt $)=\operatorname{Previous}(p f l 1)$. If it is not the first time to find overlapped $=1$ (i.e., $\mathrm{MAP}($ last_label $) \neq 0$ corresponding to overlapping_mode $=0$ ), no update is required.

\subsubsection{Equivalence}

As described before when $P(y, x) \neq 0$ and info $(x)$. line_start $=1$ and if overlap $=1$, it indicates that last_label and info $(x)$.label are equivalent. Suppose that $p l 1=$ Map (pfl1), $f l 1=N e w_{-} F_{-} L a b e l(p l 1)$, and New_F_Label(pl2) = $f l 2$. As shown in Figure 4, there are two different equivalence modes depending on $p l 2$. When the equivalence is encountered, the translation table, label-index table, and data table in the multi-layer-index should be updated to keep the consistency. When equ_mode $=1$, the update is shown in Figure 5. When equ_mode $=2$, the update is shown in Figure 6.

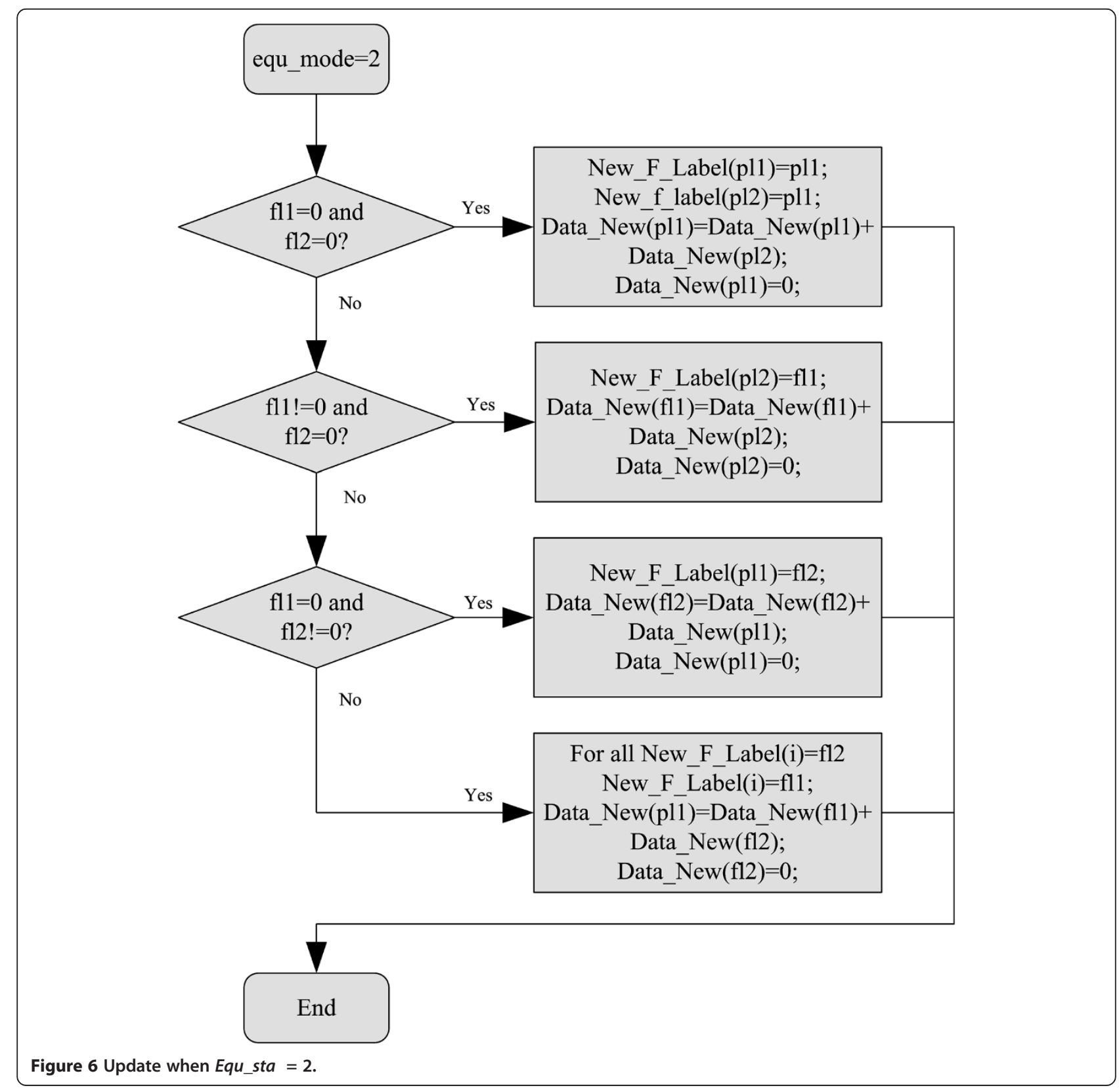




\subsubsection{Run end}

When $P(y, x)$ is found at the first pixel behind the tail of the run, the data table needs to be updated with accumulated feature data of the run. Assume the accumulated feature data are denoted as D_line, when run_end_mode $=1$, that is, the run just scanned does not connect to any runs in the previous row, Data_new $\left(n e w \_c n t\right)=D \_l i n e$. If run_end_mode $=2$, the updating is different according to $f l 1$, as it is shown in Figure 7.

After the above-mentioned update, the multi-layerindex and data table are kept up to date. The update is carried out as soon as the pixel is scanned, and no extra periods are required.

When the last pixel in the current row is scanned, Previous_F_Label is replaced with New_F_Label, and the Data_Previous is replaced with Data_New for the updating in the next row. By analyzing the translation table MAP and the previous data table Data_Previous, connected components which are completed can be found so the feature data can be passed to the next processing unit immediately, without waiting for the end of image scanning. At the end of image, a dummy row is needed for analyzing the last row in the image and initializing the memories.

\section{Hardware acceleration design}

According to the principle of proposed algorithm, the algorithm can be divided into two main blocks: (1) special cases detection and data accumulation and (2) multi-layer-index update. In the first block, the operations depending on the different positions of the scanning pixel can be processed in parallel. Meanwhile, the index and update of multi-layer-index can be accomplished by pipelining, and operations in each level of pipeline can be executed in parallel depending on the different modes (such as different equivalence modes, run end modes and overlapping modes). The proposed algorithm is implemented on Xilinx XC2V3000-6FG676

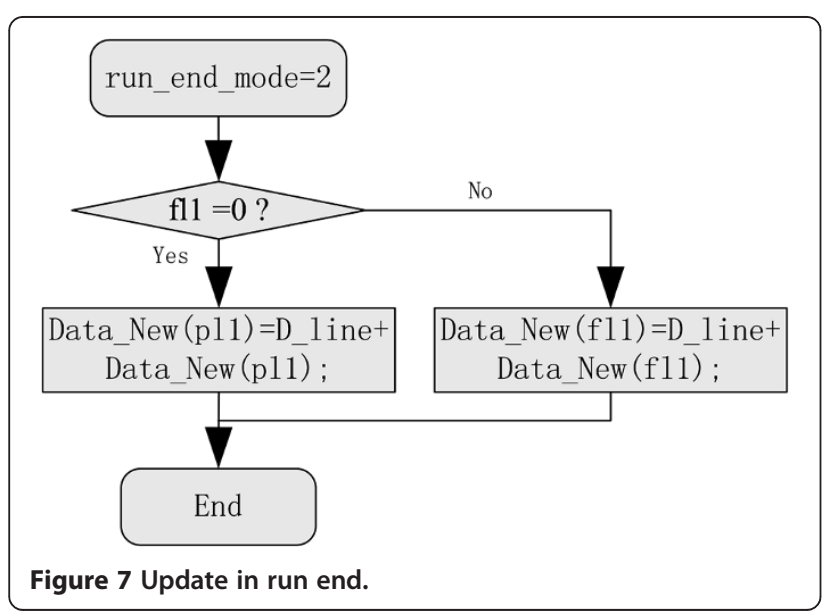

FPGA, and the architecture of hardware implementation is shown in Figure 8. As shown in Figure 8, the hardware implementation of proposed algorithm can be divided into four main blocks:

1. The row buffer block is used to store the pixel information in the previous row. It is implemented by dual-port block RAM (BRAM). Considering that the information updating of scanning pixel will conflict with the provisional label assignment at the tail of the run, two BRAMs are used alternately to store pixel information in each row.

2. The special cases detection and data accumulation block provide different flags for multi-layer-index updating; meanwhile, the feature data are also accumulated.

3. Multi-layer-index update block updates the translation table, label-index tables, and data tables, and maintains the correctness of index results in each layer. In this block, the label-index tables (such as Previous_F_Label and New_F_Label) are implemented by register array; translation table $M A P$ and data tables (such as Data_Previous, Data_New) are implemented by dual-port Block RAMs.

4. MAP and data analysis block analyze the translation table MAP and the data table Data_Previous in the scanning, then output the feature data of connected components if they are found complete.

In the implementation, three data tables, two translation tables, and two label-index tables are used in turn to achieve real-time update and analysis. We assume $\mathrm{d} 1$, $\mathrm{d} 2$, and $\mathrm{d} 3$ are three identical data tables, $\mathrm{m} 1$ and $\mathrm{m} 2$ are two identical translation tables, and i1 and i2 are two identical label-index tables before the image is scanned, they are all initialized to 0 . In the first row of image, $\mathrm{d} 1$ and $\mathrm{d} 2$ are used as Data_Previous and Data_New, m1 is used as MAP, i1 is used as Previous_F_Label, and i2 is used as New_F_Label. At the end of the first row, d2 is used as Data_Previous and d3 is used as Data_New, m2 is used as MAP, and $\mathrm{m} 1$ and $\mathrm{d} 1$ are replaced and used to extract the feature data of regions if the regions are found complete. Meanwhile, the i1 is initialized to 0 and exchanged for i2, so i2 becomes the Previous_F_Label and i1 becomes the New_F_Label in the second row. At the end of each row, the tables exchange alternately, and the analysis for completed connected components and updating of multi-layer-index can be processed in parallel.

Due to its preferable pipeline and parallel architecture, the implementation of proposed algorithm can perform real-time analysis by the original pixel clock, and no extra periods (like horizontal blanking periods in [10] and [12]) are required, which means that the time consumption of the hardware acceleration is only relevant 


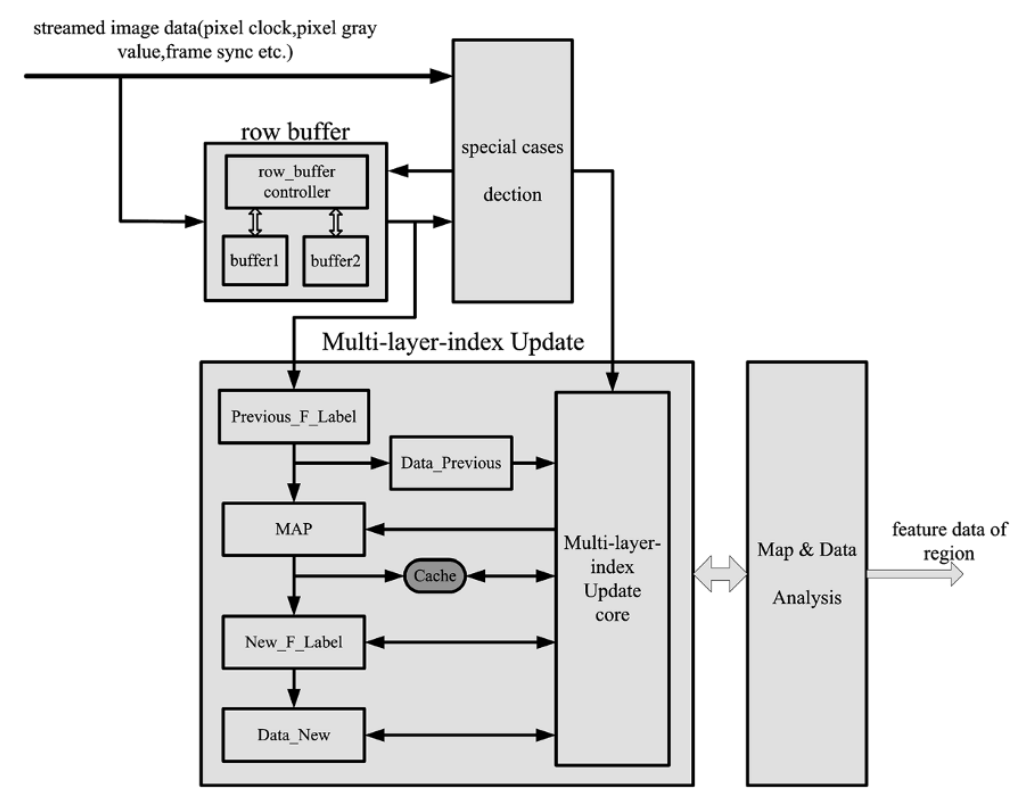

Figure 8 Architecture of hardware acceleration.

to the frequency of pixel clock and equals to the transmission period of images.

\section{Experiment results}

As aforementioned, the proposed algorithm not only runs on GPP platform, but also suits hardware acceleration in FPGA-based RTATR platform. Therefore, we verified the performance in both PC and RTATR platforms. The algorithm in [5], as an acknowledged fast connected components labeling algorithm, is selected for comparison; and running time is the key measurement in the experiment. For a more special purpose, the optimized single pass [12] algorithm which is designed for hardware implementation is also selected for comparison, and the resource utilization and processing ability are analyzed.

\subsection{Experiment in different platforms}

In the experiment, the two platforms are $\mathrm{PC}(2.5 \mathrm{GHz}+$ $2.5 \mathrm{GHz}, 2 \mathrm{G}$ memory, Windows XP OS, VC6) and our RTATR (digital signal processor, DSP: TMS320C67 13,200 $\mathrm{MHz}+$ FPGA: XC2V3000-4FG676) system. In the RTATR system, selection of processor (DSP) is limited because of the volume and power consumption constraints, the frequency of the processor is only $200 \mathrm{MHz}$, and the external bus bandwidth is ideally $400 \mathrm{MB} / \mathrm{s}$. For processor-based algorithms (like algorithm in [5]), the image can only be processed after the transmission, and the access of image data from external memory becomes the bottleneck of processing. Algorithm in [5] is performed by adding an analysis step after the CCL in the experiment. By contrast, the proposed algorithm can be implemented by FPGA in the transmission of image.
Many images have been chosen for experiment. Some of the images were acquired by the long wave infrared (IR) detector (MARS LW K508; Sofradir Company, Chatenay, Malabry, France) and without loss of generality, the others are downloaded from SIPI Image Database [13]; all the experiment images are converted to $256 \times 256$ binary images in advance. In this paper, six representative images with different connectivity complexities are selected for discussion. They are illustrated in Figure 9, in which panels a, b, and $\mathrm{c}$ are acquired from the IR detector and panels $\mathrm{d}$, e, and $\mathrm{f}$ are downloaded from the SIDBA. As shown in Figure 9, more and more connected components and equivalence occur in the Figure 9a,b,c,d,e,f. Therefore, different processing resources (e.g., buffers and processing time) are required for these images. In the comparison between the proposed algorithm and the algorithm in [5], we take these images as examples to verify the running time on different connectivity complexity condition. In the PC system, the images are loaded into the memory and then the two algorithms are performed. In RTATR system, a dedicated PCI card is used to send images to RTATR system, as same as the IR detector; the streamed data consist of image data, pixel clock, and frame synchronization sig$\mathrm{nal}$; the frequency of pixel clock is $15 \mathrm{MHz}$, and the frame rate is $100 \mathrm{~Hz}$.

As shown in Table 1, in the high-performance PC system, the algorithm in [5] can finish the CCA in less than $1 \mathrm{~ms}$; while the proposed algorithm needs more than 3 $\mathrm{ms}$ to complete the analysis of the most complex image in Figure 9. This is because the proposed algorithm contains more memory operations. But in the RTATR system, the proposed algorithm can accomplish the analysis when 


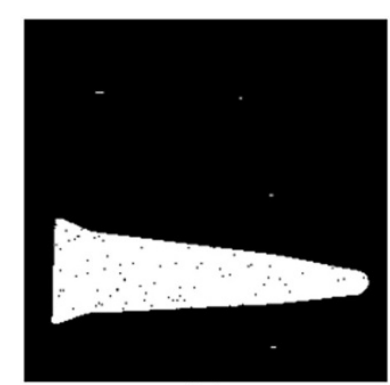

(a)

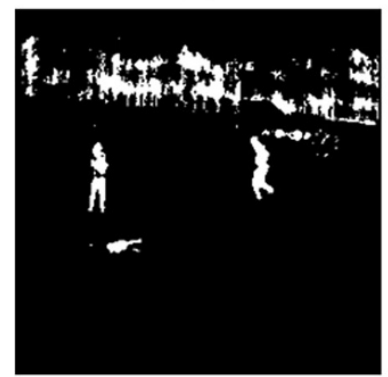

(c)

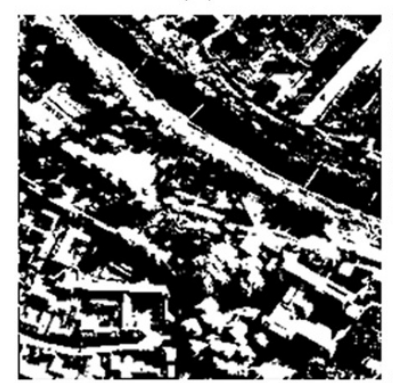

(e)

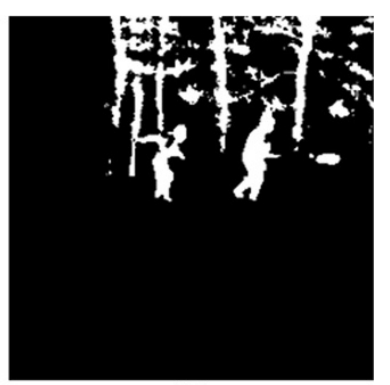

(b)

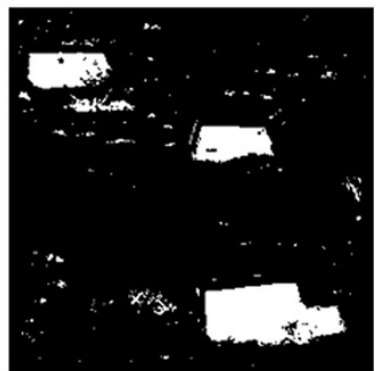

(d)

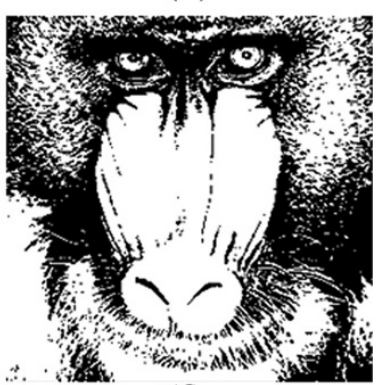

(f)
Figure 9 Experiment images. (a) Cone target, (b) two persons in forest, (c) two persons beside river, (d) truck and APCs, (e) aerial, (f) mandrill.

image is being transmitted in a raster-scan order; the processing time is a fixed value, and it is the transmission period $\left(\frac{256 \times 256}{15 \times 10^{6}} \approx 4.37 \mathrm{~ms}\right)$. By contrast, the algorithm in [5] can only process the stored image by DSP when the

Table 1 Experiment results

\begin{tabular}{lcccccc}
\hline Image & $\mathbf{C C}^{\mathbf{a}}$ & & \multicolumn{2}{c}{$\begin{array}{c}\text { Time consumption of } \\
\text { proposed algorithm }(\mathbf{m s})\end{array}$} & & \multicolumn{2}{c}{$\begin{array}{c}\text { Time consumption of } \\
\text { algorithm in [5] (ms) }\end{array}$} \\
\cline { 3 - 4 } & & PC & RTATR & & PC & RTATR \\
\hline a & 5 & 2.34 & 4.37 & & 0.32 & 22.7 \\
b & 67 & 2.81 & 4.37 & & 0.34 & 30.4 \\
c & 134 & 2.79 & 4.37 & & 0.33 & 33.7 \\
d & 220 & 2.75 & 4.37 & & 0.46 & 37.8 \\
e & 341 & 3.22 & 4.37 & & 0.91 & 62.7 \\
f & 802 & 3.33 & 4.37 & & 0.82 & 73.2 \\
\hline
\end{tabular}

${ }^{\mathrm{a}}$ Number of connected components. transmitting is over, so the time consumption is relevant to the complexity of image and exceeds the frame period (10 ms) in all the cases. This means that the algorithm is not suitable for such a RTATR platform. By accelerating the proposed algorithm on FPGA, about $5.6 \mathrm{~ms}$ is left for other algorithms after the transmission of streamed image, and real-time processing in RTATR platform is realizable.

\subsection{Hardware acceleration comparison}

In the implementation of proposed algorithm, the length of row buffers is the same size as the number of columns; in the extreme situation, there will be $M / 2$ provisional labels in a row, which means that the length of the data tables, translation tables, and label-index tables is $M / 2$. In this study, the hardware acceleration of proposed algorithm is used for analyzing the area, sum of columns, and sum of rows of pixels in each connected component and therefore, the center of each region will be obtained. Suppose the images are acquired by the aforementioned IR detector, the size of image is $256 \times$ 256 , and the streamed image data consist of 14 bits gray data, 1 bit frame synchronization, and 1 bit pixel clock, which are transmitted in raster-scan order without horizontal blanking periods. The resources and the best timing performance of pixel clock in FPGA XC2V3000-4FG676 are shown in Table 2, and the resources used in [12] is presented, too. The percentage of resources utilization of proposed algorithm is also shown in the bracket.

Comparing with existing single-pass CCA algorithm (such as hardware-implemented algorithm in [12]), our goal is to obtain the center of region, and implementation in [12] only aims at the area, therefore, the used BRAM is much more than [12]. Considering the image size is larger in [12], the occupied resource in our implementation is much more than in [12]. This is because the multi-layerindex structure and row buffer occupy more RAM and registers to store intermediate results, and more logical judgments in the indexing and updating of multi-layerindex cause more occupation of LUTs. However, the contribution of this paper is that the need of horizontal blanking periods has been eliminated for equivalence resolution. From this point of view, the consumption in our application is acceptable. By performing the CCA in the scanning, the center of each region is obtained when the

Table 2 Resource utilization

\begin{tabular}{lcc}
\hline Resource & $\begin{array}{c}\text { Proposed } \\
\text { method }\end{array}$ & $\begin{array}{c}\text { Algorithm } \\
\text { in [12] }\end{array}$ \\
\hline Used BRAMs & $13(14 \%)$ & 4 \\
Used slice flip-flops & $3,154(11 \%)$ & 600 \\
Used four-input LUTs & $4,587(16 \%)$ & 1,757 \\
Maximum clock frequency $(\mathrm{MHz})$ & 95.7 & 40.63 \\
\hline
\end{tabular}

LUTs, look-up tables. 
scanning is over. This is very important for real-time processing in systems without blanking periods (such as the IR detector in our system).

Furthermore, the maximum clock frequency of the design exceeds $90 \mathrm{MHz}$ which is more than twice the frequency which can be gotten in [12]. If the pixel clock frequency is higher (must lower than $90 \mathrm{MHz}$ ), the hardware implementation of the proposed algorithm will consume less time, and real-time CCA can be realized for larger size images.

\section{Conclusions}

In this study, a real-time single-pass connected components analysis algorithm is proposed. Compared with the existing single-pass CCA algorithms, the pixel is set as a scan unit, the run is set as a labeling unit, and the correspondence of labels in adjacent rows are managed by the multi-layer-index structure. By doing this, the equivalence can be resolved as soon as it is encountered, eliminating the need for waiting for the end of the row. Due to the preferable architecture, the algorithm can perform singlepass CCA on FPGA while the pixel is being transmitted. Experimental results indicated that the algorithm is suitable for real-time processing in the RTATR system.

\section{Competing interests}

The authors declare that they have no competing interests.

Received: 20 March 2012 Accepted: 27 March 2013

Published: 22 April 2013

\section{References}

1. H Lifeng, C Yuyan, S Kenji, A run-based two-scan labelling algorithm. IEEE. Trans. Image. Process. 17, 749-756 (2008)

2. H Lifeng, C Yuyan, S Kenji, An efficient first-scan method for label-equivalence-based labelling algorithms. Pattern. Recognit. Lett. 31, 28-35 (2010)

3. G Costantino, B Daniele, C Rita, Fast block based connected component labelling, in IEEE International Conference on Image Process (ICIP2009) (Grant Hyatt Cairo, Cairo, Grant Hyatt Cairo, Cairo, 7-10 November 2009), pp. 4061-4064

4. S Luigi Di, B Andrea, A simple and efficient connected components labelling algorithm, in International Conference on Image Analysis and Process (Venice, 27-29 September 1999), pp. 322-327

5. C Fu, C Chun-Jen, L Chi-Jen, A linear-time component-labelling algorithm using contour tracing technique. Comput. Vis. Image. Und. 93 206-220 (2004)

6. D Crookes, K Benkrid, FPGA implementation of image component labelling, in Proceedings SPIE 3844, Reconfigurable Technology: FPGAs for Computing and Applications (Boston, 20-21 September 1999), pp. 17-23

7. M Jablonski, M Gorgon, Handel-C implementation of classical component labelling algorithm, in Euromicro Symposium on Digital System Design (DSD 2004) (Rennes, 31 August to 3 September 2004), pp. 387-393

8. A Kofi, H Andrew, D Patrick, O Jonathan, A run-length based connected component algorithm for FPGA implementation, in International Conference on Field-Programmable Technology (Taibei, 8-10 December 2008), pp. 177-184

9. A Kofi, H Andrew, D Patrick, M Hongying, Accelerated hardware video object segmentation: from foreground detection to connected components labelling. Comput. Vision. Image. Und. 114, 1282-1291 (2010)

10. DG Bailey, CT Johnston, Single pass connected components analysis, in Proceedings of Image and Vision Computing (University of Waikato, Hamilton, 5-7 December 2007), pp. 282-287
11. CT Johnston, DG Bailey, FPGA implementation of a single pass connected components algorithm, in The 4th IEEE International Symposium on Electronic Design, Test and Applications (DELTA 2008) (Hong Kong, 23-25 January 2008), pp. 228-231

12. $\mathrm{M} \mathrm{Ni}$, DG Bailey, CT Johnston, Optimised single pass connected components analysis, Taibei, in International Conference on FieldProgrammable Technology (Taibei, 8-10 December 2008), pp. 185-192

13. University of Southern California, SIPI Image Database. http://sipi.usc.edu/ database/database.php?volume=misc\&image=11\#top, Accessed June 2010

doi:10.1186/1687-5281-2013-21

Cite this article as: Zhao et al:: Real-time single-pass connected components analysis algorithm. EURASIP Journal on Image and Video Processing 2013 2013:21.

\section{Submit your manuscript to a SpringerOpen ${ }^{\circ}$ journal and benefit from:}

- Convenient online submission

- Rigorous peer review

- Immediate publication on acceptance

- Open access: articles freely available online

- High visibility within the field

- Retaining the copyright to your article

Submit your next manuscript at $>$ springeropen.com 